\title{
Die wechselvolle Geschichte des Deutschen Literatur-Lexikons
}

\author{
von Bruno Jahn
}

Mit dem Band 38 erreicht die 3., völlig neu bearbeitete Auflage des von Wilhelm Kosch (I879I960) begründeten Deutschen Literatur-Lexikons. Biographisch-bibliographisches Handbuch nach etwas mehr als fünfzig Jahren das Ende des Alphabets. Der Gesamtumfang der 3. Auflage beträgt 37.402 Spalten.

\section{Die erste und zweite Auflage des Deutschen Literatur-Lexikons (1927-1930 und 1947-I958)}

Unter anderem in der Nachfolge des unmittelbar vor Ausbruch des Ersten Weltkriegs erschienenen Deutschen-Literatur-Lexikons. Biographisches und bibliographisches Handbuch mit Motivübersichten und Quellennachweisen von Herman Anders Krüger (I87I-I945) entwickelte W. Kosch sein Deutsches Literatur-Lexikon. Dessen I. Auflage mit insgesamt 3236 Spalten erschien in zwei Bänden (I927 und I930) im Max Niemeyer Verlag (Halle/Saale). In das nicht nur für Literarhistoriker im engeren Sinne, sondern auch für Journalisten, Kritiker, Dramaturgen, Bibliothekare, Buchhändler und Literaturfreunde gedachte Lexikon wurden «bei aller Knappheit der Ausführung im einzelnen» nicht nur «Dichter und Motive» aufgenommen, sondern auch «Literarhistoriker, Geschichtsschreiber, Philosophen, Kanzelredner, Verfasser bedeutender Memoiren, Briefe und Tagebücher, sowie Kulturdokumente aller Art [...], sofern sie literarisches Gepräge tragen, Anfänge von Liedern, geflügelte Worte, Zeitungen, Zeitschriften, Verleger, Gesellschaften, die sich mit Literatur befassen» (Vorwort).

Man kann wohl davon ausgehen, daß W. Kosch die Artikel größtenteils selbst verfaßte. Lebende Autoren berichten zum Teil über sich selbst; in manchen Fällen sind auch die Wohnanschriften verzeichnet. Band I (I927) umfaßt die Artikel von (Aachen〉 bis «Kosegarten, Gottfried). In Band 2 (I930), der die Artikel von «Kosegarten, Ludwig Gotthard bis «Zyrl, Christian〉 enthält, bedankt sich Kosch bei Abschluß des Lexikons «nach mehr als dreijähriger Tätigkeit» für «mannigfache Unterstützung» bei E. Arens, K. Bertsche, O. Biehler, R. Brünning, E. Gülzow, H. Häusle, W. Hammer, O. Heräus, Fr. u. E. Karg, K. K. Klein, M. Koch, O. Michaeli, H. v. Müller, Frz. Graf Pocci, E. Reinhard, A. Rosenbaum, H. Spiero, C. K. B. Susan, B. Uhlendorf, A. Waber und R. Wall. Am Ende des zweiten Bandes sind acht Spalten Berichtigungen angefügt.

Im März 1946 trug Kosch dem Francke-Verlag (Bern) die 2. Auflage seines Deutschen LiteraturLexikons an. Das Werk erschien als 2., vollständig neubearbeitete und stark erweiterte Auflage zwischen I947 und I958 in insgesamt 38 Lieferungen.

Band I: Aachen - Hasenauer. I949 (Lfg. I-9,

August I947 - August I949)

Band 2: Hasenberger - Müllner. I953

(Lfg. I0-I9, Januar I950 - Dezember 1952)

Band 3: Münch - Sparre. I956 (Lfg. 20-29,

Dezember I953 - März I956)

Band 4: Spartakus - Zyrl; Nachtrag. I958

(Lfg. 30-38, Oktober I956 - August I958)

Im Vorwort zur 2. Auflage betont Kosch, er «habe ohne einen Redaktionsausschuß und ohne einen Mitarbeiterstab die ganze Arbeit im wesentlichen allein besorgt». Aufgenommen wurden "außer den knappen Lebensläufen der Dichter, Literarhistoriker, Memoirenschreiber, Publizisten usw. aus allen Jahrhunderten, ihren eigenen Schriften und den dazu gehörigen Untersuchungen, Aufsätzen u. a., literarisch behandelte Orte und Landschaften, Liederanfänge, Stoffe und Motive, Buchtitel, Fachausdrücke usw. Auch Zeitungen, Zeitschriften und Verlage von historischer Bedeutung werden berücksichtigt.» $\mathrm{Ab}$ der 2. Auflage wurde hinter fast allen Titeln die Gattung genannt. Wertungen, wie sie in der I. Auflage gelegentlich zu finden sind so über Börne: «Leidenschaftlich bis zur schroffsten Einseitigkeit, witzig und scharf im Übermaß, Goethe gegenüber ungerecht kritisch und selbst 
mit Heine zerfallen» - wurden ab der 2. Auflage tunlichst vermieden. Kosch bedankte sich für «manchen fördernden Hinweis, manche wertvolle Ergänzung und Verbesserung», besonders aber für «die Hilfe eines ausgezeichneten Kenners der ältesten deutschen Literatur Dr. C. Minis (Rotterdam) und vor allem meines Verlegers Dr. C. L. Lang (Bern)". In den Nachtrag (Band 4, S. 35633568) wurden nur wenige Lemmata aufgenommen, darunter Imma von Bodmershof, Martin Buber und Friedrich Dürrenmatt, der oberbayerische Ort Mittenwald und das Lied «Zwoa Sternerl am Himmel».

\section{Die einbändige Ausgabe des Deutschen Literatur-Lexikons aus dem Jahr 1963}

I963 legte Bruno Berger eine einbändige Ausgabe vor (5II S.), die etwa ein Siebtel des Umfangs und ein Zehntel der Artikel der 2. Auflage des Deutschen Literatur-Lexikons bietet. Von vornherein wurden nur Verfasser und anonyme Werke aufgenommen («Maria, Mutter des Heilands` wohl versehentlich), eine Entscheidung, die bereits die Aufnahmekriterien der 3. Auflage vorwegnahm.

Der Verzicht auf Sachartikel wurde damit begründet, daß man zu ihnen in anderen Nachschlagewerken ausführlichere Informationen fände, u. a. im Reallexikon der deutschen Literaturgeschichte, das von Paul Merker und Wolfgang Stammler zwischen I925 und I93I in I. Auflage in vier Bänden herausgegeben wurde und zwischen 1958 und 1984 in einer von Werner Kohlschmidt und Wolfgang Mohr betreuten 2. Auflage erschien. Die mit der 3. Auflage (3 Bände, 1997-2003, hrsg. von Klaus Weimar, Harald Fricke und Jan-Dirk Müller in Zusammenarbeit mit anderen) angestrebte Neukonzeption, die sich schon in einer Änderung des Titels zeigt - das Titelstichwort Literaturgeschichte wurde durch Literaturwissenschaft ersetzt -, ermöglichte eine umfassende und systematische Bestandsaufnahme aller wichtigen Fachtermini der modernen Literaturwissenschaft in mehr als 900 Artikeln.

Zudem entfielen «berühmte Gedichtanfänge» und Zitate, aber auch Ortsnamen-Artikel ebenso wie die Sparte «Behandlung», unter der man z. B. in der 2. Auflage noch Artikel zu Giuseppe Garibaldi finden konnte. Übrigens erfuhr auch dessen Charakterisierung einen Wandel. Während der "auch in deutschen Gedichten und Romanen als Held benutzt[e]» Garibaldi in der I. Auflage noch als «italienischer Freischarenführer im Dienst der Einigung seiner Nation» (Sp. 549) bezeichnet wurde, wird die "Romanfigur» in der 2. Auflage - nun dem in der I. Auflage noch fehlenden Gargantua folgendzum «italienische[n] Revolutionär u. Freischarenführer im Kampf gegen Österreich u. das päpstliche Rom» (S. 6II).

Neben der Weglassung zahlreicher Lemmata, die sich in anderen Nachschlagewerken ausführlicher finden, erzwang die Beschränkung des Umfangs auch eine Auswahl unter den Autoren. Dagegen wurde das 20. Jahrhundert als Schwerpunkt erweitert um Autoren wie Theodor W. Adorno, Alfred Andersch, Ingeborg Bachmann, Heinrich Böll, Walter Benjamin, Paul Celan, Heimito von Doderer, Günter Eich, Hans Magnus Enzensberger, Louis Fürnberg, Walter Jens, Uwe Johnson, Hans Hellmut Kirst, Nelly Sachs, Traven und Martin Walser. Noch nicht wahrgenommen wurde z. B. Thomas Bernhard, der im Berichtszeitraum zwar mehrere Erzählungen und Gedichtbände, aber erst 1963 mit Frost seinen ersten Roman veröffentlichte. Im ersten Band der 3. Auflage werden Bernhard dann I8 Zeilen gewidmet sein.

Der Konzeption des "handlichen Einbänders» geschuldet ist die Kürzung zahlreicher Artikel. Waren z. B. dem Straubinger Journalisten und Schriftsteller Arthur Achleitner in der 2. Auflage noch 97 Zeilen gewidmet und wurden seine Werke einzeln aufgeführt, wird nun mit dem Hinweis, er habe «über I5O Unterhaltungsromane und Novellen» verfaßt, eine Auswahl von sechs Titeln geboten.

Erstmals wurden von Schriftstellern besprochene Schallplatten in besonderen Abschnitten aufgeführt, eine Praxis, die in der 3. Auflage unter dem Punkt «Tonträger» wesentlich erweitert wurde.

\section{Die dritte Auflage des Deutschen Literatur- Lexikons seit dem Jahr 1966}

In den I960er Jahren vereinbarte Carl Ludwig Lang (I916-I999), der bereits 1947-I958 die 2. Auflage verlegerisch betreut und I959 die Gesamtleitung der A. Francke AG übernommen hatte, mit der Witwe Annie Kosch-Lochner (I885-I976) und dem Sohn Friedrich Wilhelm Kosch (I9I2200I), daß künftige Auflagen vom Verlag veranstaltet werden. Bei der Frage nach der Gestaltung einer 3. Auflage kam Lang zu dem Entschluß, «daß dem Werk selbst und denen, die es konsultieren, am besten gedient sei, wenn sich eine Neuauflage auf den eigentlichen Kern des Deutschen Literatur-Lexikons beschränkt: auf biographische und bibliographische 
Artikel über Autoren und anonyme Werke» (Vorwort zu Bd. I, S. V). Mit dem geplanten Ausbau des Lexikons, das «über eine möglichst groBe Zahl von deutschsprachigen Autoren (und die Neulateiner des deutschen Sprachgebietes), ihre Veröffentlichungen und über anonym überlieferte Werke sachlich informieren» (S. VIII) will, war neben der Bearbeitung der einschlägigen Artikel der 2. Auflage vor allem die Aufnahme zahlreicher neuer Artikel verbunden, u. a. wegen der viel stärkeren Einbeziehung der Trivial- und Jugendliteratur. Einen erweiterten Literaturbegriff vertretend, wurden «[ü]ber die Autoren der «schönen» Literatur hinaus» auch «jene Vertreter des Geisteslebens aufgefuihrt, welche die Literatur ihrer Zeit beeinflußten oder beeinflussen. [...] Dieses Lexikon will Auskunft über alle Persönlichkeiten geben, die in Werken über die Literatur der Vergangenheit und der Gegenwart anzutreffen sind. So findet man hier also auch Theologen, Philosophen, Geschichtsschreiber, die über die Fachwissenschaft hinaus ins allgemeine Bewußtsein gedrungen sind und damit das literarische Leben befruchtet haben. [...] Verfasser von Autobiographien und Memoiren werden dann berücksichtigt, wenn sie in ihren Werken einen Beitrag zum literarischen Leben ihrer Zeit leisten. [...] Die deutschsprachigen Vertreter der germanistischen Wissenschaft sind [...] ebenfalls berücksichtigt, wobei freilich kein vollständiges Gelehrtenlexikon geboten werden soll.» (S. VI f.)

Die ursprünglich auf acht Bände projektierte 3. Auflage zeichnet sich auch durch die konsequente Aufnahme von bibliographischen Verzeichnissen aus, die in einem ersten Versuch bereits in der einbändigen Ausgabe von 1963 der Sekundärliteratur vorangestellt wurden. In den Bänden 3 und 4 sind die Beiträge zur mittelalterlichen Literatur mit Initialen, ab Band 5 alle Artikel mit einem Verfassersigel gezeichnet. Erst ab Band 30 wurden bei Aufsätzen aus Sammelbänden und Zeitschriften die Seitenzahlen angegeben. In welchem Umfang die 3. Auflage ausgebaut und erweitert wurde, zeigt sich darin, daß beispielsweise der Buchstabe A in der 2. Auflage 203 Verfasserartikel aufwies, während im Band I der 3. Auflage 495 Lemmata geboten werden; im Ergänzungsband I kamen beim Buchstaben A 945 weitere Artikel hinzu (jeweils ohne Verweise).

Die Gewinnung der Herausgeber und Mitarbeiter der 3. Auflage übernahm vornehmlich der
Cheflektor des Verlags, Helmut Bender. Eine eigene Redaktion mit Sitz in Freiburg/Br. wurde eingerichtet. Im Frühjahr 1976 schuf der Verlag eine neue Arbeitsgruppe; eine "eigene Redaktion und Mitarbeiter für die Abfassung der zahlreichen Kleinartikel» wurden als «unerläßlich» gesehen (Vorwort zu Bd. 6, S. V), was mit sich brachte, daß der Hauptteil der Artikel von festen Mitarbeitern des Verlags verfaßt wurde, worauf in den folgenden Bänden regelmäßig hingewiesen wird. Diesen Verlagsmitarbeitern wurden für ihre Arbeit am Deutschen Literatur-Lexikon in der Schweizerischen Landesbibliothek und der Stadt- und Universitätsbibliothek Bern Arbeitsplätze zur Verfügung gestellt.

Der erste Band der 3., völlig neu bearbeiteten Auflage erschien in vier Lieferungen (Dezember I966 - März 1968). Die nächsten Bände, die aus «arbeitstechnischen Gründen» nicht mehr in Lieferungen ausgegeben wurden, folgten I969, I97I, I972; Band 5 erschien aus finanziellen Gründen erst nach einer längeren Pause. «Um den Zeitraum zwischen dem Erscheinen des vierten und des fünften Bandes etwas zu verringern, entschloß sich der Verlag, Band fünf in zwei Hälften aufzuteilen. Die erste Hälfte (bis Spalte 448) konnte im Januar 1977 ausgegeben werden» (S. V). Mit dem Erscheinen der zweiten Hälfte im Sommer 1978 lag der Band vollständig vor. Im Vorwort zu Band 6 wird auf die «Notwendigkeit einer Straffung» hingewiesen und der Umfang der 3. Auflage auf is Bände festgelegt, was sich jedoch als nicht machbar erwies. Zwar soll das Lexikon weiterhin über eine «möglichst groBe Zahl von deutsch-sprachigen Autoren (und die Neulateiner des deutschen Sprachgebiets) und ihre Veröffentlichungen sowie über anonym überlieferte Werke sachlich informieren» (S. V), «im Mittelpunkt des Lexikons stehen [aber] die Verfasser und anonym überlieferten Werke, die zur Literatur im engeren Sinne gehören» (S. VI). «Die Vita bringt jeweils Fakten, auf Wertung wird verzichtet. Die selbständigen Buchveröffentlichungen werden jeweils in chronologischer Folge in der Regel vollständig aufgefuihrt. Die Sekundärliteratur bringt je nach ihrem Umfang eine kleine oder größere Auswahl» (S. V).

Nach der Übernahme der A. Francke AG (Bern) durch den K. G. Saur Verlag (München; 2006 vom Verlag Walter de Gruyter, Berlin, übernommen) 1990 erschien das Lexikon «unverändert in der bewährten Form». Eine Modernisierung der 
technischen Abläufe trug zur Beschleunigung des Erscheinungsrhythmus bei. Ergänzungsbände ( $a b$ I994) zu den Buchstaben A - F (Bd. I-5, I968I978) sollten die ungleiche Informationsdichte ausgleichen. Die Zahl der Ergänzungsbände wurde schließlich auf sechs erhöht, weil Ergänzungen über den Buchstaben $\mathrm{F}$ hinaus sich als notwendig erwiesen. Der sechste und letzte Ergänzungsband (1999) enthält Ergänzungen bis einschließlich Buchstabe R. Die Ergänzungen erstrecken sich nur auf zusätzlich aufgenommene Autoren und Anonyma; Ergänzungen zu Artikeln in den ersten I3 Bänden wurden nicht geliefert.

\section{Die Herausgeber, Redaktionen und Mitar- beiter des Deutschen Literatur-Lexikons}

Die Bände I und 2 der 3. Auflage wurden von Bruno Berger (Freiburg/Br.) und Heinz Rupp (Basel) gemeinsam herausgegeben. Auf den Titelblättern der Bände 3 und 4 wird das von Wilhelm Kosch begründete Lexikon als «fortgefuihrt von Bruno Berger» bezeichnet. Als Herausgeber der Älteren Abteilung zeichnet ab Band 3 Heinz Rupp. Anders verhält es sich bei der Neueren Abteilung, deren Herausgeber wechseln: Band 4: Friedrich Gaede (17. Jahrhundert) und Detlev Steffen (I8. Jahrhundert; beide Halifax, Kanada), Band 5: Hildegard Emmel (Storrs/CT). Die Bände 6 bis Is und die Ergänzungsbände I und II gaben Heinz Rupp (Ältere Abteilung, später Mittelalter [bis ca. I500]) und Carl Ludwig Lang (Neuere Abteilung, später Neuzeit [ca. I5Oo bis zur Gegenwart]) heraus, die Bände I6 bis I9 und die Ergänzungsbände III bis VI Hubert Herkommer (Mittelalter) und Carl Ludwig Lang (Neuzeit), die Bände 20 bis $29 \mathrm{Hu}-$ bert Herkommer (Mittelalter) und Konrad Feilchenfeldt (ca. I50o bis zur Gegenwart). Mit dem Übergang der Redaktion von Zürich nach München übernahmen ein Wissenschaftlicher Beirat (Wolfgang Achnitz, Lutz Hagestedt, Mario Müller, Claus-Michael Ort, Reimund B. Sdzuj) die Herausgeberaufgaben und Bruno Jahn die redaktionelle Leitung. Der Beirat regte an, die biographischen Anteile der Beiträge informativer zu gestalten, die Werksbibliographien möglichst vollständig zu präsentieren und auch die Verzeichnisse der Sekundärliteratur, Tonträger, Überlieferung etc. anzureichern. Diese neue Qualität erklärt u. a., weshalb für die Buchstaben W (Weiß) bis Z insgesamt neun Bände seit dem Jahr 2010 erschienen sind (Bde. 30 bis 38 ).
Damit ist die 3. Auflage des Deutschen LiteraturLexikons nach etwas mehr als fünfzig Jahren beinahe abgeschlossen. Dieser lange Zeitraum erscheint für die Fülle der zusammengetragenen Informationen gerechtfertigt, vor allem weil nun ein in sich geschlossenes Lexikon zur biographischbibliographischen Literaturgeschichte zur Verfügung steht, das nicht nur im deutschen Sprachraum seinesgleichen sucht. Naturgemäß zieht eine solche Erscheinungsdauer Nachträge und Aktualisierungen nach sich, die mit sechs Ergänzungsbänden in den Jahren von I994 bis 1999 bereits vorgelegt worden sind. Es werden nach dem Erscheinen des letzten Bandes des Alphabets (Bd. 38) noch drei Nachtragsbände und die notwendigen Register zur Erschließung des Lexikons folgen.

Die Mitarbeiter der bisherigen Bände waren:

Achnitz, Wolfgang (Münster/Westf.): Bd. 32

Altenhöfer, Florian (München, später Leipzig):

Bde. $3 \mathrm{I}-38$

Bauer, Werner M. (Innsbruck): Bd. 6

Bender, Helmut (Freiburg/Br.): Bde. $5-8$

Berger, Bruno (Freiburg/Br.): Bde. I-5

Bigler (später Bigler-Marschall), Ingrid (Bern, später Zürich): Bde. 7-I6, ErgBde. I-III,

Bde. I7-30

Bircher, Martin (Wolfenbüttel, später Cologny):

Bde. 6-I3, ErgBd. I, Bd. 2 I

Blinn, Hansjürgen (Saarbrücken, später St. Ingbert, Kirkel): Bde. 9, II-I2, I4-I6, ErgBd. V, Bd. I8, ErgBd. VI, Bde. $2 \mathrm{I}-22,25-30,35$

Blühm, Elger (Bremen): Bd. 6

Bölhoff, Reiner (Freiburg/Br.): Bd. 5

Bonawitz, Achim (Detroit/MI): Bd. 9

Bucheli, Roman (Zürich): Bd. I7, Erg-

Bde. IV-V, Bd. I8, ErgBd. VI

Buchhester, Dörthe (Stralsund): Bde. 3I-32, 38

Cleave, Jan (München): Bd. 35

Cobbs, Alfred (Detroit/MI): Bde. 8-II

Crowhurst, Griseldis (Freiburg/Br.): Bde. I-2

Daemmrich, Horst (Detroit/MI, später Philadelphia/PE): Bde. 6-9, II-I3

Dewitz, Hans-Georg (Frankfurt/M., später Eschborn): Bde. 6-IO, I2-I4, I7

Eben, Erdmuthe-Annika (München, später Augsburg): Bde. $30-35$

Evans, Tamara S. (Flushing/NY): Bde. 8, Io

Fasbender, Christoph (Chemnitz): Bde. 34, 37

Faulhaber, Uwe (Detroit/MI): Bde. 6-II

Feger, Robert (Freiburg/Br.): Bd. 5 
Fehr, Karl (Frauenfeld): Bd. 6

Feldges, Mathias (Basel): Bde. 2-4

Flury, Theres (Zürich): Bde. I9-2I

Foidl, Sabina (München): Bde. 30-33, 35-38

Garber, Klaus (Osnabrück): Bd. I2

Goff, Penrith (Detroit/MI): Bde. 6-I6

Guthke, Karl S. (Cambridge/MA): Bd. 7

Haase, Donald (Detroit/MI): Bde.IO-II

Hagestedt, Lutz (Rostock): Bde. 32, 35, 38

Hay, Gerhard (München): Bd. 7

Hees, Anke (Zürich): ErgBde. V-VI, Bd. I8

Heiduk, Franz (Würzburg): Bde. 7-I6, Erg-

Bde. I-III, Bde. I7-I8

Heinemann, Erich (Hildesheim): Bd. Io

Hertz, Hans W. (Hamburg): Bde. 6-7

Hille, Peter (Freiburg/Br.): Bde. $2-5$

Hoffmann, Dirk O. (Hamilton/NY): Bd. 9

Hohl, Siegmar (Freiburg/Br.): Bd. 2

Hopp, Doris (Frankfurt/M.): Bd. 6

Hostettler, Christian (Belp): Bd. 9, ErgBd. II

Ingen, Ferdinand J. van (Amsterdam): Bd. 6

Jahn, Bruno (München): Bde. 30-38

Jenuwine, Daniel (Detroit/MI): Bde. I4-IS

Kanz, Claudia (Chemnitz): Bd. 35

Kenosian, David (Philadelphia/PE): Bd. I4

Kieser, Harro (Frankfurt/M.): Bde. 5-II, I6-I9

Kirsten, Wulf (Weimar): Bde. 7-I6, ErgBde.

I-III, Bde. I7-32, 34

Klingner, Jacob (Berlin): Bde. 32, 34, 37-38

Knedlik, Manfred (Nürnberg, später Landshut,

Neumarkt): Bde. 2I-33, 36-38

Königs, Nadine (München): Bde. 30-3I

Kosch, Friedrich Wilhelm (Graz): Bd. 9

Kully, Max Rolf (Basel): Bde. 2-4

Lang, Margrit (Bern): Bde. I4-I6, ErgBde. I-III, Bde. $17-30$

Larkin, Edward (Philadelphia/PE, später Durham/NH): Bde. II-I2

Lieberwirth, Christian (Chemnitz): Bde. 32, 34

Linder, Heinz-Peter (Bern): Bde. I-5

Lokai, Deborah (Detroit/MI): Bde. I2-I3

Luserke, Matthias (Saarbrücken): Bd. I5

Malm, Mike (München): Bde. 30-38

Mannack, Eberhard (Heikendorf): Bd. 6

Meyer, Gerd (Rauschenberg): Bd. I4

Meyer, Margret (Freiburg/Br.): Bde. 3-4

Michel, Christoph (Frankfurt/M., später Freiburg/Br.): Bde. 6, IO, I4, 2I-30, 32, 34-38

Mieder, Wolfgang (Burlington/VT): Bd. II

Müller, Mario (Stralsund, später Chemnitz, Hildesheim): Bde. $3 \mathrm{I}-38$
Müller, Reinhard (Bern, später Zürich):

Bde. 6-I5, ErgBde. I-II, Bd. I6, ErgBd. III, Bd. I7, ErgBde. IV-V, Bd. I8, ErgBd. VI,

Bde. $19-29$

Murphy, Marylin (Philadelphia/PE): Bde. II-I2

Ochsenbein, Peter (Basel, später St. Gallen): Bde. 2-6, 8

Parr, Rolf (Dortmund): Bd. I7

Pörnbacher, Hans (Wildsteig): Bde. I5-29, 33, 36,38

Rebeggiani, Matteo (Chemnitz): Bde. 32-34

Reinhold, Konrad (Chemnitz): Bde. 32-34

Röll, Walter: Bd. 5

Roth, Maria (Detroit/MI): Bde. 6-II

Ruge, Nikolaus (Trier): Bd. 33

Ruprecht, Robert (Burgdorf/Kt. Bern): Bde. 6-7

Sassen, Frederike von (München): Bde. 3I-32

Schantz, Randy (Detroit/MI): Bde. I2-I3

Schmid, Ursula (Basel): Bd. 4

Schmid-Cadalbert, Christian (Basel): Bd. II

Schneider, Cordelia (Freiburg/Br.): Bd. 5

Schnidrig, Benita (Bern): Bd. I9

Schultz, Hartwig (Frankfurt/M.): Bde. 6-8

Schumacher, Erika (Basel): Bde. 5, 7

Sdzuj, Reimund B. (Greifswald): Bde. 3I-36, 38

Seehafer, Klaus (Diepholz): Bd. 7

Spellerberg, Gerhard (Berlin): Bd. 7

Spreizer, Christine (Philadelphia/PE): Bd. I3

Stridde, Christine (Zürich): Bd. 38

Stüssi, Anna (Bern): Bde. 6-I6, ErgBde. I-III, Bde. I7-I9

Suchomski, Joachim (Basel): Bd. 5

Taegert, Werner (Bamberg): Bd. Io

Tekolf, Olive (Leipzig): Bde. 3I-32

Tischler, Maria (München): Bde. 26-38

Tischler, Paul (München): Bde. 24, 26-38

Ulrich, Margot (Düsseldorf): Bd. Io

Webb, Kathy M. (Philadelphia/PE): Bd. II

Weschenfelder, Anke (Wetzikon, später Steg/Kt.

Zürich, Fischenthal, Sennhof): Bde. 2I-30

Weydt, Günther (Münster/Westf.): Bd. 6

Wilhelm, Joanne (Philadelphia/PE): Bd. II

Wilke, Klaus-Peter (Freiburg/Br.): Bd. 5

Willmitzer, Christoph (Berlin): Bd. 3I

Zapf, Volker (München): Bde. 30-38

Zimmermann, Heinz: Bde. I-2

Die Redaktion der Bände lag bei: Hanspeter Bennwitz (I, I. Lfg.), Siegmar Hohl (I, ab 2. Lfg.; 2, bis zum Schluß des Buchstabens B), Helmut 
Bender unter Mitwirkung von Cordelia Schneider (3), Helmut Bender mit Cordelia Schneider und Klaus-Peter Wilke (4), Helmut Bender mit KlausPeter Wilke (5), Carl Ludwig Lang (6-I9, ErgBde. I-III), Ingrid Bigler-Marschall und Reinhard Müller (20-29), Bruno Jahn (30-38).

Band I: Aal - Bremeneck. Bern: Francke, I968 Band 2: Bremer - Davidis. Bern: Francke, 1969 Band 3: Davidis - Eichendorff. Bern: Francke, I97I

Band 4: Eichenhorst - Filchner. Bern: Francke, I972

Band 5: Filek - Fux. Bern: Francke, I978

Band 6: Gaa - Gysin. Bern: Francke, I978

Band 7: Haab - Hogrebe. Bern: Francke, I979

Band 8: Hohberg - Kober. Bern: Francke, 198I

Band 9: Kober - Lucidarius. Bern: Francke, I984

Band io: Lucius - Myss. Bern: Francke, I986

Band II: Naaff - Pixner. Bern: Francke, I988

Band I2: Plachetka - Rilke. Bern: Francke, I99o

Band I3: Rill - Salzmann. Bern: Francke, I99I

Band I4: Salzmesser - Schilling. Bern: Francke, 1992

Band I5: Schilling - Schnydrig. Bern: Saur, 1993

Ergänzungsband I: A - Bernfeld. Bern: Saur, 1994

Ergänzungsband II: Bernfeld - Christen. Bern/ München: Saur, I994

Band I6: Schobel - Schwaiger. Bern/München: Saur, 1996

Ergänzungsband III: Christener - Fowelin. Bern/München: Saur, I997

Band I7: Schwalb - Siewert. Bern/München: Saur, 1997

Ergänzungsband IV: Fraenkel - Hermann. Bern/ München: Saur, I997

Ergänzungsband V: Hermann - Lyser. Bern/ München: Saur, 1998

Band I8: Siff - Spoerri. Bern/München: Saur, I998

Band I9: Spohn - Sternaux. Bern/München: Saur, 1999

Ergänzungsband VI: Maag - Ryslavy. Bern/ München: Saur, 1999

Band 20: Sternbach - Streißler. Bern/München: Saur, 2000

Band 2r: Streit - Techim. Zürich/München: Saur, 200I

Band 22: Tecklenburg - Tilisch. Zürich/

München: Saur, 2002
Band 23: Tikla - Trystedt. Zürich/München:

Saur, 2003

Band 24: Tsakiridis - Ursinus. Zürich/München:

Saur, 2004

Band 25: Ursprung - Vöhringer. Zürich/

München: Saur, 2005

Band 26: Völckel - Wagner. Zürich/München:

Saur, 2006

Band 27: Wagner - Walser. Zürich/München:

Saur, 2007

Band 28: Walsh - Wedegärtner. Zürich/

München: Saur, 2008

Band 29: Wedekind - Weiß. Berlin/New York:

De Gruyter, 2009

Band 30: Weiß - Werdum. Berlin/New York:

De Gruyter, 20I0

Band 3r: Werenberg - Wiedling. Berlin/Boston:

De Gruyter, 2012

Band 32: Wiedmann - Willisen. Berlin/Boston:

De Gruyter, 20I3

Band 33: Willius - Wircker. Berlin/Boston: De Gruyter, 20I4

Band 34: Wirdig - Wol. Berlin/München/

Boston: De Gruyter, 20I5

Band 35: Wolf - Worbs. Berlin/München/

Boston: De Gruyter, 20I6

Band 36: Worch - Zasius. Berlin/Boston: De Gruyter, 2017

Band 37: Zass - Zimdar. Berlin/Boston: De Gruyter, 2018

Band 38: Zimmer - Zyx. Berlin/Boston: De Gruyter, 2019

\section{Parallel- und Tochterunternehmungen zum Deutschen Literatur-Lexikon}

Seit dem Jahr 2000 erscheint das Deutsche LiteraturLexikon. Das 20. Jahrhundert, zunächst von Carl Ludwig Lang, ab Band 2 (200I) von Konrad Feilchenfeldt und ab Band I4 (20I0) von Lutz Hagestedt herausgegeben.

Zwischen 20II und 20I6 erschien das Deutsche Literatur-Lexikon. Das Mittelalter. Autoren und Werke nach Themenkreisen und Gattungen, herausgegeben von Wolfgang Achnitz, in acht Bänden mit weit mehr als 5000 Artikeln (I Das geistliche Schrifttum von den Anfängen bis zum Beginn des I4. Jahrhunderts - II Das geistliche Schrifttum des Spätmittelalters - III Reiseberichte und Geschichtsdichtung - IV Lyrik und Dramatik - V Epik (Vers - 
Strophe - Prosa) und Kleinformen - VI Das wissensvermittelnde Schrifttum bis zum Ausgang des I4. Jahrhunderts - VII Das wissensvermittelnde Schrifttum im I5. Jahrhundert - VIII Nachträge, Chronologie und Register). Gegenüber alphabetisch sortierten Nachschlagewerken ähnlichen Inhalts liegt sein Mehrwert darin, "dass es das mittelalterliche Schrifttum in seinem Formenreichtum und in seiner inhaltlichen Vielfalt systematisch in einzelnen Bänden präsentiert und innerhalb dieser Bände nach aktuellem wissenschaftlichen Stand chronologisch zu ordnen versucht» (Schlußwort, Bd. 8, S. VII). Zudem bieten die Bände jeweils sachkundige Einleitungen.
Die Online Reference Deutsches Literatur-Lexikon Online (seit 20I7) macht die umfangsreichsten Nachschlagewerke zur deutschen Literatur erstmals systematisch elektronisch nutzbar: Das Deutsche Literatur-Lexikon und die flankierenden Reihen (Deutsches Literatur-Lexikon. Das 20. Jahrhundert, Deutsches Literatur-Lexikon. Das Mittelalter) sind einzigartig in ihrer umfassenden Darstellung. Mehr als 70000 Artikel sind digital aufbereitet und mit differenzierten Kriterien gezielt durchsuchbar. Der Datenbestand wird kontinuierlich durch bibliographische Daten der Germanistik Online aktualisiert und erweitert. Updates finden zweimal jährlich statt. 
